\title{
X-ray detection of the substellar twin 2MASS J11011926-7732383 AB
}

\author{
B. Stelzer and G. Micela
}

INAF - Osservatorio Astronomico di Palermo, Piazza del Parlamento 1, 90134 Palermo, Italy

e-mail: stelzer@astropa.unipa.it

Received 27 March 2007 / Accepted 5 June 2007

\section{ABSTRACT}

\begin{abstract}
Context. 2MASS J11011926-7732383 AB (hereafter 2M 1101 AB), located in the Cha I star forming region, is a rare wide-separation brown dwarf binary. Being spatially resolvable in many wavebands, it is a unique target for studying the properties of substellar twins. Aims. Here, we exploit the coeval pair $2 \mathrm{M} 1101 \mathrm{AB}$ to examine the influence of physical parameters (mass, bolometric luminosity and effective temperature) on X-ray emission from substellar objects.

Methods. We determine the X-ray properties of 2M 1101 A and B using XMM-Newton and Chandra observations.

Results. The spatial resolution of XMM-Newton is not sufficient to separate contributions from the two components in the binary. The X-ray source detected with XMM-Newton has a column density compatible with the infrared extinction of component A. On the other hand, the binary is resolved with Chandra, and the bulk of the X-ray emission is clearly associated with the photospherically cooler component B. These apparently contradictory results point at strong variability of $2 \mathrm{M} 1101$ 's X-ray emission. Combined with previous sensitive X-ray observations from low-mass members of Cha I, we find a decline of X-ray luminosity with decreasing (sub)stellar mass that is typical for star forming regions.

Conclusions. 2M $1101 \mathrm{~B}$ is the coolest (spectral type M8.25) and least massive brown dwarf of Cha I detected in X-rays so far. It is also among the youngest ( 1 Myr) substellar Cha I members, and therefore relatively luminous. Most bona fide brown dwarfs of Cha I have remained below the sensitivity limits of available X-ray observations, because of their low luminosity associated with higher age.
\end{abstract}

Key words. X-rays: stars - stars: coronae - stars: activity - stars: low-mass, brown dwarfs - stars: pre-main sequence stars: individual: 2MASS J 11011926-7732383 AB

\section{Introduction}

The binary brown dwarf 2MASS J11011926-7732383 AB (hereafter 2M 1101) was discovered by Luhman (2004b) during a search for substellar objects in the Cha I star forming region. Upon its discovery it was the first known wide brown dwarf binary, and as such it constitutes an important discriminant for the formation mechanisms of brown dwarfs: the wide binary separation of 1.44" (corresponding to $240 \mathrm{AU}$ at the distance of $168 \mathrm{pc}$; Whittet et al. 1997; Wichmann et al. 1998) practically excludes the ejection scenario that predicts a maximum separation of $\sim 10 \mathrm{AU}$ for substellar binaries expelled from a multiple system without being disrupted (Bate et al. 2002).

According to Luhman (2004b), the two components in the 2M1101 system have spectral types M7.25 and M8.25, and bolometric luminosities of 0.02 and $0.0062 L_{\odot}$, respectively. Figure 1 shows the position of 2M 1101 in the HR diagram, together with other low-mass Cha I members from the catalogs of Luhman (2004a) and Comerón et al. (2004). The 2M1101 binary (large filled circles in Fig. 1) is coeval with an age of $\sim 1$ Myr on the evolutionary tracks by Chabrier et al. (2000). The probability for the two being a chance projection has been estimated by Luhman (2004b) to be extremely low $\left(5 \times 10^{-5}\right)$. The individual masses of the two components according to the Chabrier et al. models are $0.05 M_{\odot}$ and $0.025 M_{\odot}$ for $2 \mathrm{M} 1101 \mathrm{~A}$ and $\mathrm{B}$, respectively, well below the transition to the substellar regime. The physical parameters of 2M 1101 are summarized in Table 1.
Evidently, 2M 1101 is a unique target for studying the properties of substellar twins, i.e. coeval brown dwarfs of slightly different effective temperature, luminosity, and mass. Its wide separation allows to resolve the two components with presentday instrumentation in many wavelength regimes, including the soft X-ray band.

$\mathrm{X}$-ray emission from late-type stars is a well-known signature of magnetic activity (Rosner et al. 1985). Young pre-main sequence (pre-MS) stars show much higher levels of X-ray luminosity than evolved stars in open clusters and in the field (e.g. Feigelson \& Montmerle 1999). The empirical saturation level for the X-ray luminosity of active stars is $\log \left(L_{\mathrm{x}} / L_{\text {bol }}\right) \approx-3$, but for given $L_{\mathrm{bol}}$ the spread reaches about 2 dex below this threshold. According to recent observations the $L_{\mathrm{x}}-L_{\mathrm{bol}}$ relation seems to hold even in the substellar mass regime (Preibisch et al. 2005b; Grosso et al. 2007).

The universality of the $L_{\mathrm{x}}-L_{\mathrm{bol}}$ relation can not be taken for granted, especially in view of the fact that pre-MS stars as well as brown dwarfs are fully convective and the standard solar-like $\alpha \Omega$-dynamo, which requires a transition between a radiative and a convective layer, is not expected to work. Alternative field generating mechanisms involving turbulence have been invoked to explain X-ray emission from the fully convective lowest mass stars at the bottom of the MS and from brown dwarfs (Durney et al. 1993). The effects of a change in the dynamo mechanism are both difficult to predict and observationally poorly constrained. While there is consensus that the X-ray luminosity declines towards the substellar boundary, it is unclear which role 


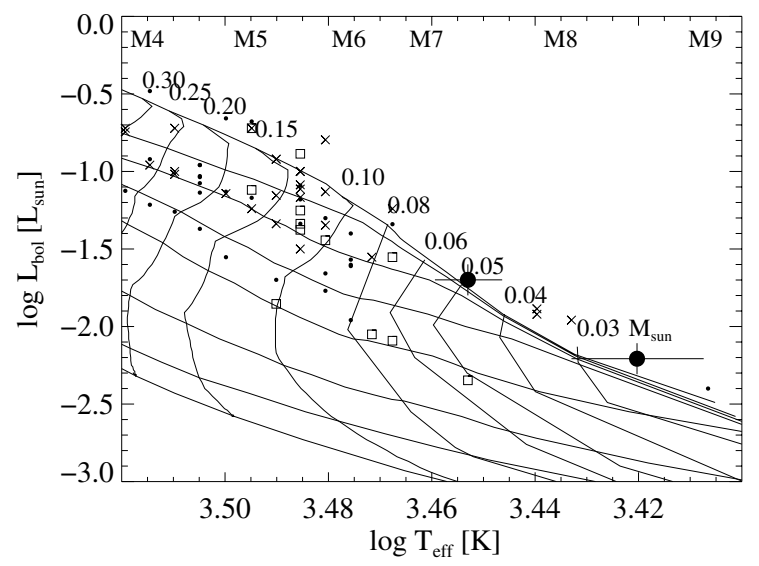

Fig. 1. HR diagram for low-mass members of Cha I; data from Luhman (2004a) and Comerón et al. (2004); models from Baraffe et al. (1998) for $M \geq 0.1 M_{\odot}$ and from Chabrier et al. (2000) for $M<0.1 M_{\odot}$. The tracks are labeled by their mass, and the substellar limit corresponds to $0.07 M_{\odot}$. Isochrones represent from top to bottom log Age [yrs] = 6.0, 6.3, 6.5 , 6.7, 7.0 7. 7.5, 8.0 and 9.0. Plotting symbols: crosses - Cha I members detected in X-rays (Stelzer et al. 2004; Feigelson \& Lawson 2004; Robrade \& Schmitt 2007); squares - Cha I members not detected in X-rays; small circles - Cha I members not observed in X-rays. large filled circles - the brown dwarf binary 2M $1101 \mathrm{~A}$ and 2M $1101 \mathrm{~B}$ (stellar parameters from Luhman 2004b), whose X-ray data are discussed in this paper. The least massive brown dwarf in Cha I, OTS 44 (see Luhman et al. 2004), is off the plotted range to the right, and not detected in X-rays.

Table 1. Physical parameters of the 2M 1101 system relevant to this study; data from Luhman (2004b).

\begin{tabular}{llrrrr}
\hline \hline Component & SpT & $\begin{array}{r}T_{\text {eff }} \\
{[\mathrm{K}]}\end{array}$ & $\begin{array}{r}L_{\text {bol }} \\
{\left[L_{\odot}\right]}\end{array}$ & $\begin{array}{r}A_{J} \\
{[\mathrm{mag}]}\end{array}$ & $\begin{array}{r}A_{\mathrm{V}}^{(1)} \\
{[\mathrm{mag}]}\end{array}$ \\
\hline $\mathrm{A}$ & $\mathrm{M} 7.25$ & 2838 & 0.020 & 0.45 & 1.6 \\
$\mathrm{~B}$ & $\mathrm{M} 8.25$ & 2632 & 0.0062 & $0(<0.2)$ & $0(<0.7)$ \\
\hline
\end{tabular}

(1) From $A_{J}$ using the extinction law of Rieke \& Lebofsky (1985).

different physical parameters besides $L_{\text {bol }}$ (e.g. rotation, effective temperature, mass, age, binarity) play in the efficiency of $\mathrm{X}$-ray production. Especially, the effective temperature might be crucial as it determines the ionization state of the atmosphere. Mohanty et al. (2002) have argued that the electrical resistivity in the nearly neutral atmospheres of very low-mass (VLM) dwarfs may be enhanced to the point that no efficient coupling of matter and magnetic field is possible, preventing magnetic activity. A possible direct dependence between X-ray luminosity and $T_{\text {eff }}$ has recently been pointed out by Stelzer et al. (2006).

Whatever is the origin of the $L_{\mathrm{x}}-L_{\mathrm{bol}}$ relation, it ensues that the chance for an X-ray detection is higher for young brown dwarfs than for less luminous evolved ones. Indeed, in recent years deep X-ray observations with Chandra and XMM-Newton in star forming regions have turned up an increasing number of $\mathrm{X}$-ray detections among young brown dwarfs (e.g. Preibisch et al. 2005b; Grosso et al. 2007).

Cha I is relatively nearby, and therefore it was among the first star forming regions with a well-defined brown dwarf population. For the median age of Cha I members ( 2 Myr), the substellar limit corresponds to spectral type M6 to M6.5 (Baraffe et al. 1998). The sample of brown dwarfs and candidate brown dwarfs in Cha I identified in an $\mathrm{H} \alpha$ emission line survey (Comerón et al. 2000), yielded the first X-ray detection of a substellar object with ROSAT (Neuhäuser \& Comerón 1998). In a more recent
Table 2. Observing log for the X-ray observations of 2M 1101.

\begin{tabular}{l|rr}
\hline \hline Mission/Instrum. & XMM/EPIC-pn & Chandra/ACIS-S \\
\hline Target & Cha I Field G & 2M 1101-7732 \\
PI & M. Güdel & B.Stelzer \\
Obs-ID & 0152460301 & 6396 \\
Date [UT] & $2002-04-09 @ 09: 39: 20$ & $2006-05-15 @ 04: 36: 46$ \\
Exposure [ks] & 34 & 40 \\
\hline
\end{tabular}

XMM-Newton observation of the southern part of Cha I, that includes all $13 \mathrm{H} \alpha$ emitting brown dwarfs and brown dwarf candidates from the survey of Comerón et al. (2000), the X-ray detection fraction for the faint " $\mathrm{ChaH} \alpha$ " objects was increased from $6 / 13$ to $9 / 13$ thanks to the higher sensitivity and higher spatial resolution of XMM-Newton with respect to ROSAT (Stelzer et al. 2004). Further X-ray studies with Chandra and XMM-Newton concentrating on the northern part of the Cha I cloud have been presented by Feigelson \& Lawson (2004) and Robrade \& Schmitt (2007).

The X-ray detected and non-detected objects from these surveys are distinguished by different plotting symbols in Fig. 1 (see figure caption). They have been placed in the HR diagram using the effective temperatures and luminosities given by Luhman (2004b). With respect to previous estimates for the physical parameters, some of the objects that used to be considered brown dwarfs have now moved across the substellar boundary into the stellar domain; see Sect. 4 for more details. It is evident from Fig. 1 that the X-ray detection rate beyond the substellar boundary is rather low. According to Luhman's compilation of physical parameters and the Chabrier et al. (2000) models, only three bona-fide brown dwarfs and two objects at the border line were detected in X-rays: ChaH $\alpha$ 1, ChaH $\alpha$ 7, CHSM-17173, ISO-217, and $\mathrm{ESOH} \alpha 566$. On the other hand, all Cha I members with $M>0.2 M_{\odot}$ that were in the field-of-view of one of the abovementioned X-ray observations were detected.

All three X-ray detected bona-fide brown dwarfs in Cha I seem to be very young, located above the $1 \mathrm{Myr}$ isochrone. The two components of the binary 2M1101 AB have similarly young age and bracket $\mathrm{ChaH} \alpha 1, \mathrm{ChaH} \alpha$, and CHSM-17173 in terms of mass and effective temperature. Here we discuss recent Chandra and XMM-Newton observations of 2M 1101. The observations and data analysis are described in Sect. 2, and Table 2 provides the observing log. While XMM-Newton provides higher collecting area, only Chandra has the potential to separate the binary and to compare the X-ray properties of the two components of this benchmark substellar twin. The X-ray properties of $2 \mathrm{M} 1101 \mathrm{~A}$ and B are discussed in Sect. 3. In Sect. 4 the results are combined with the published X-ray data for other low-mass Cha I members. This allows us to investigate the dependence of $L_{\mathrm{x}}$ on physical parameters such as bolometric luminosity, effective temperature and mass for the brown dwarf population in Cha I, and to compare it to similar studies in the Orion and Taurus star forming regions.

\section{Observations and data analysis}

\subsection{Chandra}

2M 1101 was observed for $40 \mathrm{ks}$ with Chandra's Advanced CCD Imaging Spectrometer for Spectroscopy (ACIS-S); see Weisskopf et al. (2002) for details on the satellite and its instruments. The data analysis was carried out using the CIAO 
software package ${ }^{1}$ version 3.3.0.1. We started our analysis with the level 1 events file provided by the Chandra X-ray Center (CXC). In the process of converting the level 1 events file to a level 2 events file we performed the following steps. A correction for the charge transfer inefficiency has been applied. We removed the pixel randomization which is automatically applied by the CXC pipeline in order to optimize the spatial resolution. We filtered the events file for event grades (retaining the standard $A S C A$ grades 0, 2, 3, 4, and 6), and applied the standard good time interval (GTI) file. Events flagged as cosmic rays were retained after inspection of the images revealed that a substantial number of source photons erroneously carry this flag. Since the positional accuracy is particularly important to our observation we also checked the astrometry for any known systematic aspect offset using the CIAO aspect calculator ${ }^{2}$. This tool confirms that no offset larger than $1^{\prime \prime}$ is present.

Source detection was performed with the WAVDETECT algorithm (Freeman et al. 2002) on an image with $0.25^{\prime \prime}$ pixel size centered on the position of the primary $2 \mathrm{M} 1101 \mathrm{~A}$ and excluding photons with energy outside the $0.5-8 \mathrm{keV}$ band. WAVDETECT correlates the data with a mexican hat function to search for deviations from the background. This method is well suited for separating closely spaced point sources. We used wavelet scales between 1 and 8 in steps of $\sqrt{2}$.

We detect one X-ray source at the position of the binary. Despite an obvious offset with respect to the near-IR position (Fig. 2), we are confident that this source can be identified with component $\mathrm{B}$. This assertion is supported by a check of the astrometry using the only other nearby X-ray star in the field; for details see Sect. 3. There is no detected source associated with 2M $1101 \mathrm{~A}$, but a possible enhancement of the count rate may evidence weak X-ray emission from this component.

For further analysis we extracted photons assigned to 2M $1101 \mathrm{~B}$ from a circular region of $1.0^{\prime \prime}$ radius centered on the X-ray position. That area includes $91 \%$ of the PSF. Photons assigned to $2 \mathrm{M} 1101 \mathrm{~A}$ are extracted from a circular region of $0.5^{\prime \prime}$ radius centered on its near-IR position. This latter region includes $66 \%$ of the PSF. We note, that the extraction areas of components A and B overlap, but there are no photons in the overlapping region; cf. Fig. 2. The background is very low (cf. Sect. 3) and can be neglected.

\subsection{XMM-Newton}

$2 \mathrm{M} 1101$ is in the field-of-view of a $30 \mathrm{ks} X M M-$ Newton $^{3}$ observation. We extracted the data taken with the European Photon Imaging Camera pn CCD (EPIC/pn) from the XMM-Newton archive. The observations were performed in full-frame mode employing the medium filter.

Starting from the pipeline products, we filtered the events file for pixel patterns (retaining only singles and doubles), edge effects at the boundary of individual CCD chips, events outside the field-of-view, and near bad pixels. We also eliminated the lowest pulse height channels to further reduce the noise. We searched for times of high background by selecting the uncontaminated time intervals with a filter that maximizes the signal-to-noise as a function of the count rate across the whole detector array

\footnotetext{
${ }^{1}$ CIAO is made available by the $\mathrm{CXC}$ and can be downloaded from http://cxc.harvard.edu/ciao/download-ciao-reg.html

2 See http://asc.harvard.edu/ciao/threads/ arcsec_correction

3 The satellite and its instruments are described in a Special Issue of A\&A (2001, Vol. 365).
}

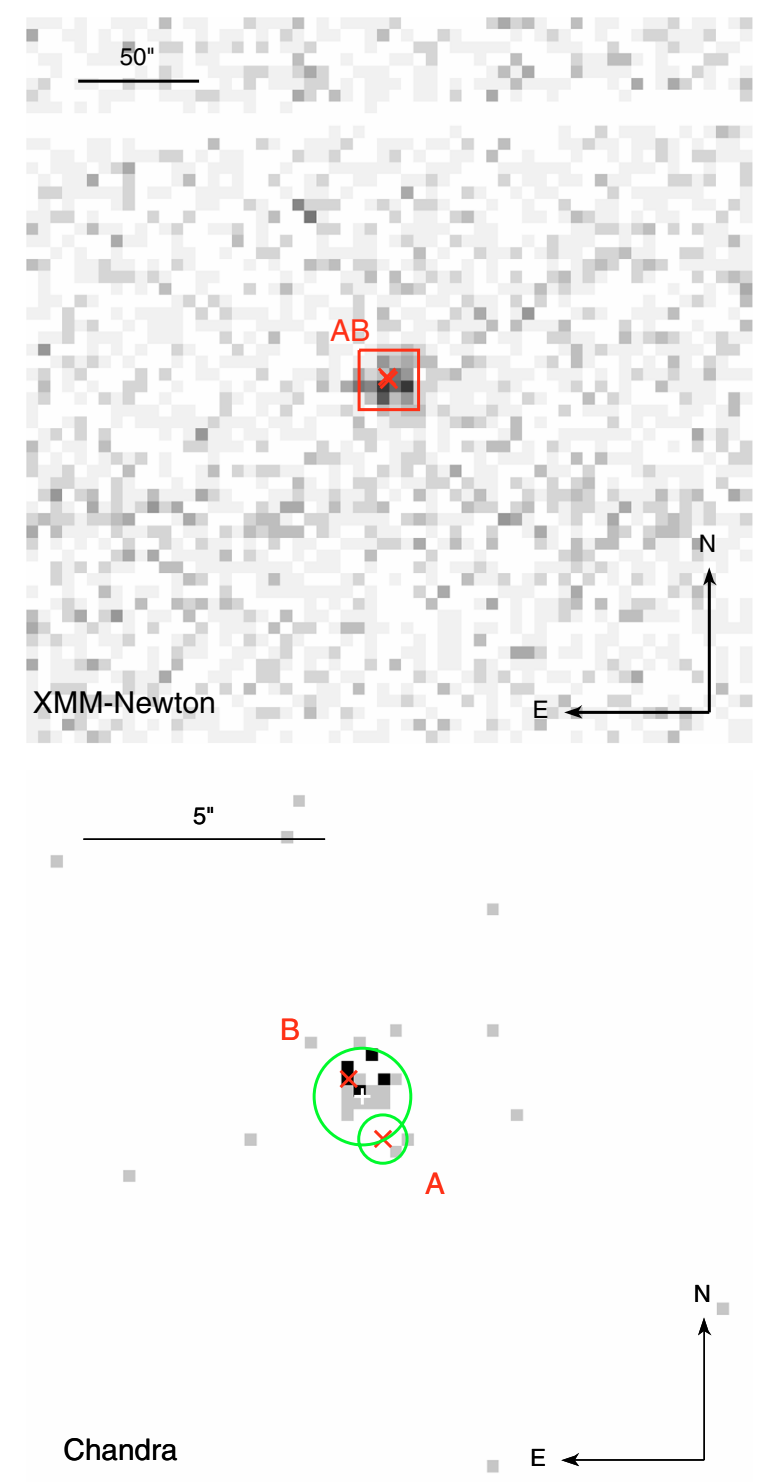

Fig. 2. X-ray images of $2 \mathrm{M} 1101$ in the $0.5-8 \mathrm{keV}$ band: XMM-Newton EPIC/pn in the top panel with $5^{\prime} \times 5^{\prime}$ image size, $5^{\prime \prime}$ pixel size and offaxis angle of $6.6^{\prime}$ for $2 \mathrm{M} 1101$. The white horizontal line near the top of the EPIC/pn image is a CCD gap. The red square indicates the position and size of the Chandra ACIS-S image shown in the bottom panel. Image size for Chandra is $24.6^{\prime \prime} \times 24.6^{\prime \prime}$, bin size is $0.25^{\prime \prime}$, and $2 \mathrm{M} 1101$ is on-axis. X-points in the XMM-Newton and in the Chandra image denote the near-IR positions given by Luhman (2004b) for the individual components in the binary system. The circles in the Chandra image represent the extraction areas; see text in Sect. 2.1.

and the exposure time. The data turned out to be widely unaffected by background flaring, with the exception of a brief time interval near the middle of the observation that was removed from the GTIs. This way the effective observing time is reduced to $28.6 \mathrm{ks}$. In the subsequent analysis only photons that arrived within the GTIs were considered.

$2 \mathrm{M} 1101 \mathrm{AB}$ is clearly detected, but as the spatial resolution of EPIC is well above the binary separation, the two components can not be resolved (see Fig. 2). We extracted source photons from a circle with $30^{\prime \prime}$ radius around the position of $2 \mathrm{M} 1101 \mathrm{~A}$. According to the XMM-Newton User's Handbook ${ }^{4}$ this radius

\footnotetext{
4 See http://xmm.vilspa.esa.es/external/ xmm_user_support/documentation/uhb/XMM_UHB.html
} 
Table 3. X-ray properties of $2 \mathrm{M} 1101 \mathrm{~A}$ and B. For XMM-Newton we list results for the time-average, flare (f) and quiescent (q) state.

\begin{tabular}{|c|c|c|c|c|c|c|c|c|c|}
\hline Component & $\begin{array}{c}\Delta_{\mathrm{ix}}^{(1)} \\
{\left[{ }^{\prime \prime}\right]} \\
\end{array}$ & Net counts ${ }^{(2,3)}$ & $\begin{array}{l}\text { Net rate }{ }^{(2,3)} \\
\text { [cts/s] }\end{array}$ & $\begin{array}{r}N_{\mathrm{H}} \\
{\left[10^{21} \mathrm{~cm}^{-2}\right]} \\
\end{array}$ & $\begin{array}{r}k T \\
{[\mathrm{keV}]}\end{array}$ & $\chi_{\text {red }}^{2}$ & (d.o.f.) & $\begin{array}{r}f_{\mathrm{x}}^{(2,4)} \\
{\left[\mathrm{erg} / \mathrm{cm}^{2} / \mathrm{s}\right]}\end{array}$ & $\begin{array}{r}\log L_{\mathrm{x}}{ }^{(2,4)} \\
{[\mathrm{erg} / \mathrm{s}]}\end{array}$ \\
\hline \multicolumn{10}{|c|}{ XMM-Newton EPIC/pn } \\
\hline $\mathrm{A}+\mathrm{B}$ & & $148 \pm 13.2$ & $(5.7 \pm 0.5) \times 10^{-3}$ & $2.8_{1.7}^{4.4}$ & $0.9_{0.6}^{1.4}$ & 0.9 & (13) & $3.0 \times 10^{-14}$ & 29.0 \\
\hline (f) & & $73.5 \pm 9.6$ & $(1.0 \pm 0.1) \times 10^{-2}$ & $3.8_{0.7}^{6.1}$ & $0.9_{0.5}^{2.0}$ & 1.6 & (6) & $6.2 \times 10^{-14}$ & 29.3 \\
\hline (q) & & $74.5 \pm 9.7$ & $(4.0 \pm 0.5) \times 10^{-3}$ & $3.1_{1.7}^{6.4}$ & $0.6_{0.4}^{1.1}$ & 1.0 & (12) & $2.2 \times 10^{-14}$ & 28.8 \\
\hline \multicolumn{10}{|c|}{ Chandra ACIS-S ${ }^{(5)}$} \\
\hline A & 1.1 & $2 \pm 2.7$ & $(5.1 \pm 6.8) \times 10^{-5}$ & $=2.5$ & $=0.9$ & & & $<1.4 \times 10^{-15}$ & $<27.6$ \\
\hline $\mathrm{B}$ & 0.5 & $21 \pm 5.6$ & $(5.3 \pm 1.4) \times 10^{-4}$ & $=0.0$ & $=0.9$ & & & $2.1 \times 10^{-15}$ & 27.8 \\
\hline
\end{tabular}

(1) Error of the offset derived from the uncertainty of the X-ray position is $\approx 0.1^{\prime \prime}$ for ACIS;

(2) in $[0.5,8.0] \mathrm{keV}$;

(3) errors are computed using the Gehrels description $(1+\sqrt{N+0.75})$.

(4) fluxes and luminosities are corrected for encircled PSF fraction and for absorption; the upper limit is based on the $95 \%$ confidence value derived as described in the text.

${ }^{(5)}$ Numbers preceeded by "=" are assumed values, see text.

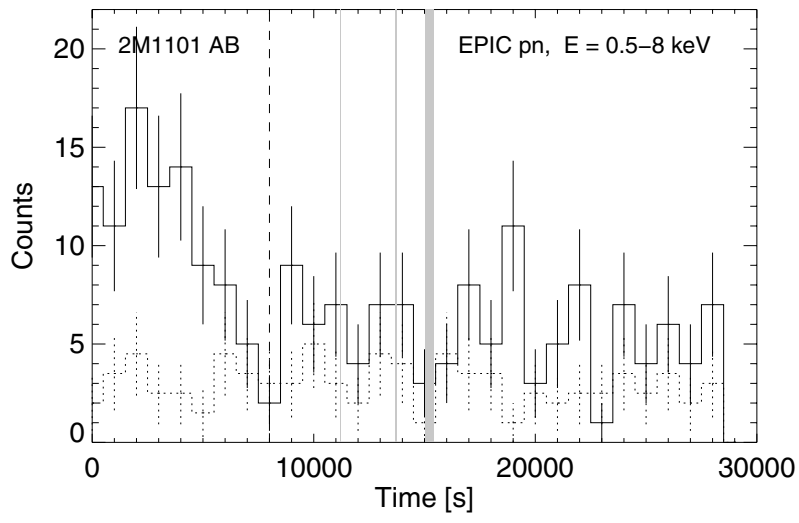

Fig. 3. XMM-Newton EPIC/pn lightcurve of 2M $1101 \mathrm{AB}$ : solid line Signal from the position of $2 \mathrm{M} 1101 \mathrm{AB}$ comprising source and background, dotted line - Background from source-free regions as described in the text. The grey shades indicate "bad time intervals" removed from the data, and the dashed line marks the point chosen to distinguish flaring and quiescent state.

comprises $\sim 80 \%$ encircled energy. Background photons were extracted from two $30^{\prime \prime}$ circles positioned on the same CCD chip, at the same distance from the read-out node as the source extraction area and avoiding the read-out strip of a nearby bright $\mathrm{X}$-ray source. For the spectral analysis we generated a response matrix and ancilliary response file using standard XMM-Newton Science Analysis System tools. The spectrum was binned to a minimum of 15 counts per bin and analysed in the XSPEC v.11.3.0 environment.

\section{Results}

We begin the discussion of the results with the XMM-Newton data, where the statistics are higher. The EPIC/pn lightcurve extracted from the position of $2 \mathrm{M} 1101 \mathrm{AB}$ is shown in Fig. 3 together with the area-scaled background lightcurve. A phase of enhanced source signal at the beginning of the observation is distinguished. The background is constant throughout the observation, and can not be responsible for this flare-like feature. A Kolmogorov-Smirnov (KS) test for the source+background lightcurve yielded a probability for variability $P>99 \%$, while no significant variability is detected with the KS test in the background-only lightcurve. The mean net source count rate

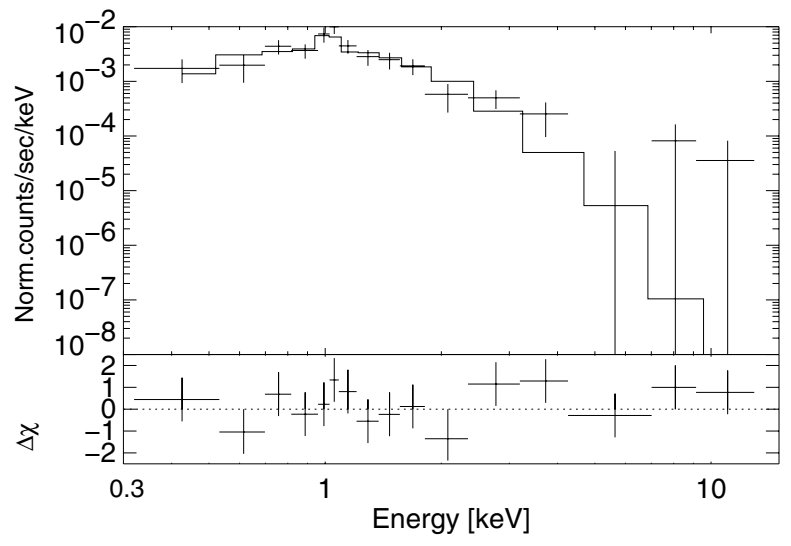

Fig. 4. Time-averaged XMM-Newton EPIC/pn spectrum of the unresolved binary system $2 \mathrm{M} 1101 \mathrm{AB}$ : data, bestfit model and residuals.

of the combined emission from $2 \mathrm{M} 1101 \mathrm{AB}$ measured with EPIC/pn is given in Table 3. We further split the observation in a flaring and a quiescent part; the dividing line is indicated in Fig. 3. Net source count rates for the two activity states are also listed in Table 3.

The time-averaged EPIC/pn spectrum is displayed in Fig. 4. Albeit stellar coronae are known to be composed of a multitemperature plasma, in the case of poor statistics the X-ray spectra are often approximated fairly well by a one-temperature thermal model describing a plasma in collisional ionization equilibrium with a photoabsorption term (e.g. Getman et al. 2005). We adopt the APEC code (Smith et al. 2001) with the WABS absorption as implemented in XSPEC (v.11.3). For the elemental abundances of the X-ray emitting plasma we use the values presented by Maggio et al. (2007). This set of abundances was determined from fitting the X-ray spectra of a sample of X-ray bright pre-MS stars in Orion. The major deviation with respect to photospheric abundances (e.g. Anders \& Grevesse 1989; Asplund et al. 2005) is the low iron abundance and the high $\mathrm{Ne} / \mathrm{Fe}$ ratio, a pattern that has repeatedly been found in stellar coronae (see e.g. review by Güdel 2004). The bestfit of such a model to the observed time-averaged EPIC/pn spectrum of $2 \mathrm{M} 1101 \mathrm{AB}$ has $\chi_{\text {red }}^{2}=0.9$ with 13 degrees of freedom. The bestfit parameters for average, flaring and quiescent spectrum are listed in Table 3 . During the phase of high activity at the beginning of the observation the temperature is higher than during the 


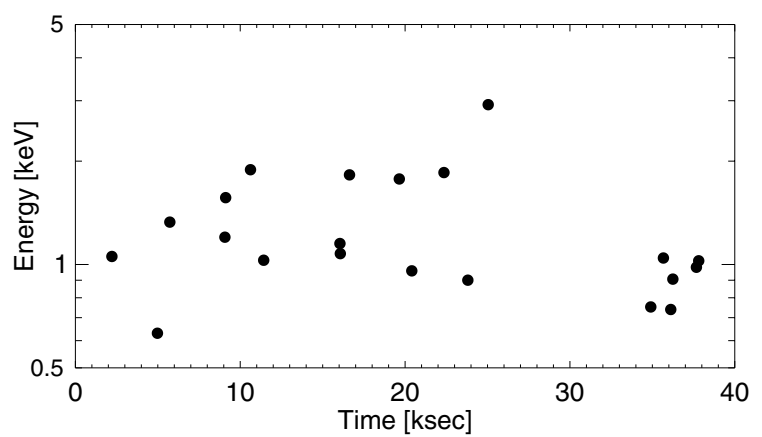

Fig. 5. Photon energy vs. arrival time for the Chandra X-ray source associated with 2M $1101 \mathrm{~B}$. There are no bad-time intervals.

following quiescent phase (although not statistically significant), as is typical for stellar flares.

The spectral fit of the time-averaged EPIC/pn spectrum yields an absorbing column $N_{\mathrm{H}}$ (cf. Table 3 ) that corresponds to a near-IR extinction $A_{J} \approx 0.29 \ldots 0.84 \mathrm{mag}$ using the gas-todust conversion law derived by Vuong et al. (2003) for pre-MS stars in $\rho$ Oph. This range of $A_{J}$ includes both the observational $90 \%$ error of $N_{\mathrm{H}}$ and the uncertainty in the $N_{\mathrm{H}} / A_{J}$ relation. The near-IR extinction estimated this way is consistent with the $o b$ served $A_{J}$ of $2 \mathrm{M} 1101 \mathrm{~A}$ but higher than the value of $2 \mathrm{M} 1101 \mathrm{~B}$ (cf. Table 1). This result suggests that the emission is dominated by component $\mathrm{A}$.

Obviously, there is no way to separate the contributions of $2 \mathrm{M} 1101 \mathrm{~A}$ and $\mathrm{B}$ to the X-ray spectrum and lightcurve observed with XMM-Newton, and we resort to the Chandra data where the two components can be resolved. Contrary to the preliminary conclusion from the XMM-Newton spectrum, the secondary, 2M $1101 \mathrm{~B}$, is much closer to the X-ray source detected with WAVDETECT than the primary, 2M 1101 A. Figure 2 suggests a shift of the X-ray image with respect to the near-IR positions in the south-west direction. Other than centering the $\mathrm{X}$ ray source onto the position of $2 \mathrm{M} 1101 \mathrm{~B}$, such a shift would clearly identify 2M 1101 A with the nearby 'cluster' of two photons. The image can not be checked for a systematic boresight error because there is not a sufficient number of X-ray bright sources with optical/IR counterparts. However, another X-ray emitting Cha I member (CS Cha) is nearby (3.7' off-axis). We compared the position of the corresponding X-ray source to the optical position of CS Cha, and found an offset of only 0.06 ". Therefore, we are confident that no significant shifts are present in the X-ray image.

With a total of 21 net counts in the extraction area the Chandra source associated with 2M 1101 B is too weak for spectral analysis. However, some information can be gained from Fig. 5, where we plot the energy versus the arrival time of the individual photons in the extraction circle of $2 \mathrm{M} 1101 \mathrm{~B}$. The source has a remarkably hard spectrum, with more than half of the photons having energies in excess of $1 \mathrm{keV}$. The median photon energy of $2 \mathrm{M} 1101 \mathrm{~B}$ in the $0.5-8 \mathrm{keV}$ band is $1.06 \pm 0.17 \mathrm{keV}$, where the uncertainty was estimated with the Maritz-Jarrett method as described by Hong et al. (2004). From nearby source free regions we estimate that the expected number of background photons in the extraction area of $2 \mathrm{M} 1101 \mathrm{~B}$ is only 0.22 in the $0.5-8 \mathrm{keV}$ interval. Therefore, the background can be neglected in the evaluation of the flux in this energy band.

Clearly, the detection of 2 photons with energies between $0.5-8 \mathrm{keV}$ from $2 \mathrm{M} 1101 \mathrm{~A}$ is significantly higher than the number of contaminating photons from the X-ray source associated with component B. The source extraction area of $2 \mathrm{M} 1101 \mathrm{~A}$ comprises $3 \%$ of all photons in the PSF of $2 \mathrm{M} 1101 \mathrm{~B}$, i.e. 0.66 photons. There are 0.05 background counts expected for the same energy band in the extraction area of $2 \mathrm{M} 1101 \mathrm{~A}$. Considering further the fact that the two photons are coincident with the position of $2 \mathrm{M} 1101 \mathrm{~A}$, they probably both originate from this brown dwarf. Nevertheless, local fluctuations of the background can not be excluded, and we prefer to assign an upper limit to the count rate of this component. The "observed" number of photons from 2M 1101 A extrapolated across the full PSF is 3 counts. Using the prescription by Kraft et al. (1991) for Poisson-distributed counting data we derive a $95 \%$ confidence upper limit of $\sim 8$ counts for $2 \mathrm{M} 1101 \mathrm{~A}$.

The source fluxes of 2M $1101 \mathrm{~A}$ and B given in Table 3 were evaluated with PIMMS ${ }^{5}$ on basis of their individual near-IR extinctions and the temperature of $0.9 \mathrm{keV}$ from the EPIC/pn spectrum. The sum of the fluxes of both components estimated this way from the Chandra observation is almost a factor 10 below the time-averaged $X M M-N e w t o n$ flux, and a factor of $>5$ below the quiescent XMM-Newton flux. If instead the $N_{\mathrm{H}}$ value measured from the EPIC/pn spectrum is adopted for the spectral model in PIMMS, the combined ACIS-S flux of 2M $1101 \mathrm{~A}$ and $\mathrm{B}$ increases by a factor of 2 . Source variability may be in part responsible for the remaining discrepancy with respect to the XMM-Newton flux.

Indeed, in Fig. 5 there is a striking absence of photons between $\sim 25 \ldots 35 \mathrm{ks}$ from the start of the observation. To quantify the suspicion of source variability we applied a test proposed by Preibisch \& Zinnecker (2002): first, we determine the time interval $\Delta t$ in which 2 photons are expected to be detected under the assumption of a constant signal corresponding to the time averaged count rate. Then, the maximum number of counts $N_{\max }$ in any such interval $\Delta t$ across the observation is computed. The Poisson probability that $N_{\max }$ is a statistical fluctuation is given by $P=1-\sum_{k=0}^{N_{\max }-1} \mathrm{e}^{-2} \frac{2^{k}}{k !}$. We find $P=0.016$. Therefore, $2 \mathrm{M} 1101 \mathrm{~B}$ was probably variable during the Chandra observation.

\section{Discussion}

\subsection{Comparison of XMM-Newton and Chandra results}

At first sight, the Chandra detection of the secondary component and the non-detection (or extreme weakness) of the primary in the 2M 1101 system seems to contradict the $L_{\mathrm{x}}-L_{\mathrm{bol}}$ relation. However, one must take account of the fact that the $\mathrm{X}$-ray photons are subject to absorption. Luhman (2004b) measured negligible extinction for $2 \mathrm{M} 1101 \mathrm{~B}$ but $A_{J}=0.45 \mathrm{mag}$ for $2 \mathrm{M} 1101 \mathrm{~A}$. If we assume that both objects have the same $\mathrm{X}$-ray flux and spectrum with temperature of $\sim 1 \mathrm{keV}$, we can estimate the effect of the absorbing column onto the observed spectrum. With PIMMS we found that the count rate of the unabsorbed $\left(N_{\mathrm{H}}=0\right) \mathrm{X}$-ray source $2 \mathrm{M} 1101 \mathrm{~B}$ corresponds to a flux of $2.1 \times 10^{-15} \mathrm{erg} / \mathrm{cm}^{2} / \mathrm{s}$. If the column density was $N_{\mathrm{H}}=$ $2.5 \times 10^{21} \mathrm{~cm}^{-2}$ (equivalent to the observed $A_{J}$ of $2 \mathrm{M} 1101 \mathrm{~A}$ ), a total of $7-8$ counts would be expected in the extraction area of $2 \mathrm{M} 1101 \mathrm{~A}$, in contrast to the 2 counts observed. In order to shrink the count rate to the observed level, both a lower temperature $(\approx 0.6 \mathrm{keV})$ and a higher extinction $\left(N_{\mathrm{H}} \approx 5 \times 10^{21} \mathrm{~cm}^{-2}\right)$ must be hypothesized for $2 \mathrm{M} 1101 \mathrm{~A}$. This is, however, unconsistent with the XMM-Newton spectrum. We conclude, that during the Chandra observation the X-ray flux of 2M $1101 \mathrm{~A}$ was

\footnotetext{
5 The Portable Interactive Multi-Mission Simulator (PIMMS) is accessible at http: //asc . harvard.edu/toolkit/pimms . jsp
} 
significantly lower than that of the photospherically cooler secondary $2 \mathrm{M} 1101 \mathrm{~B}$.

Furthermore, the XMM-Newton spectrum suggests that the dominant X-ray emitter during the XMM-Newton observation was $2 \mathrm{M} 1101 \mathrm{~A}$, while during the Chandra observation clearly the dominant X-ray emitter was 2M $1101 \mathrm{~B}$, and consequently both objects must be strongly variable in X-rays. Using the results given in Table 3 this leads to the conclusion that the combined X-ray luminosity of $2 \mathrm{M} 1101 \mathrm{AB}$ can vary by at least a factor of 10. An alternative interpretation is that the dominant X-ray emitter was $2 \mathrm{M} 1101 \mathrm{~B}$ during both observations but its $N_{\mathrm{H}}$ is higher than the near-IR value. In this latter case, the Chandra flux can be estimated using the $N_{\mathrm{H}}$ obtained from the $X M M-N e w t o n$ spectrum. This flux is $4.4 \times 10^{-15} \mathrm{erg} / \mathrm{cm}^{2} / \mathrm{s}$, a factor of two higher than for negligible extinction. However, it is still a factor of seven lower than the XMM-Newton flux. Therefore, in this scenario the X-ray emission of 2M $1101 \mathrm{~B}$, again, must be variable. Furthermore, the $N_{\mathrm{H}}$ value from the $X M M$-Newton spectrum corresponds to a near-IR extinction of $A_{J} \approx 0.5 \mathrm{mag}$, and this would shift $2 \mathrm{M} 1101 \mathrm{~B}$ upward in the HR diagram. As a consequence, it would no longer be coeval with 2M1101 A, an unlikely scenario.

We caution that the above considerations rely on the gas-todust extinction law presented by Vuong et al. (2003). Deviations from the standard extinction law have been seen in other samples of young stars (cf. Feigelson et al. 2005). Such discrepancies can arise from errors in the X-ray spectral model or in the photometry, or they may represent untypical environmental conditions.

\subsection{X-ray emission from brown dwarfs in Cha I}

To put 2M $1101 \mathrm{~A}$ and B into the context of the X-ray properties of low-mass stars, we revisit published X-ray data for the Cha I star forming region. Studies of the X-ray population of Cha I with XMM-Newton and Chandra have been presented by Stelzer et al. (2004), Feigelson \& Lawson (2004), and Robrade \& Schmitt (2007). We resume here the results on the VLM stars and brown dwarfs based on the membership catalog of Luhman (2004a) with additional 9 members discovered by Comerón et al. (2004). Previous estimates for the physical parameters had placed some of the objects which now appear to be stars into the brown dwarf regime. This was due to a combination of (i) slightly different spectral types assigned, (ii) a different SpT / $T_{\text {eff }}$ conversion and (iii) the use of other evolutionary models. Luhman (2004a) has shown that for the Cha I population the models by Baraffe et al. (1998) and Chabrier et al. (2000) provide the best agreement with observational constraints, including higher-mass T Tauri stars up to $M=1 M_{\odot}$. We use these models and the physical parameters from Luhman (2004a) and Comerón et al. (2004), to estimate individual masses for all X-ray detected Cha I members from the above-mentioned publications.

Then we re-inspected these X-ray source lists for consistency with the updated membership list. Upper limits for nondetections were not discussed in the X-ray surveys, and we will not consider them here. The assessment of upper limits is difficult because of their dependence on the spectral shape and, more importantly, the (unknown) extinction. We verified that, for the observations discussed here, these upper limits range roughly between $\log L_{\mathrm{x}}[\mathrm{erg} / \mathrm{s}]<28.5$ and $<27.5$. All X-ray detected Cha I members are included in the X-ray catalogs. For the faint objects from Robrade \& Schmitt (2007), we computed $L_{\mathrm{X}}$ from the listed count rates and conversion factor, assuming a distance of $160 \mathrm{pc}$. The examined fields include 75 detected and 20 undetected Cha I members. All non-detections have masses below $0.2 M_{\odot}$, i.e. the

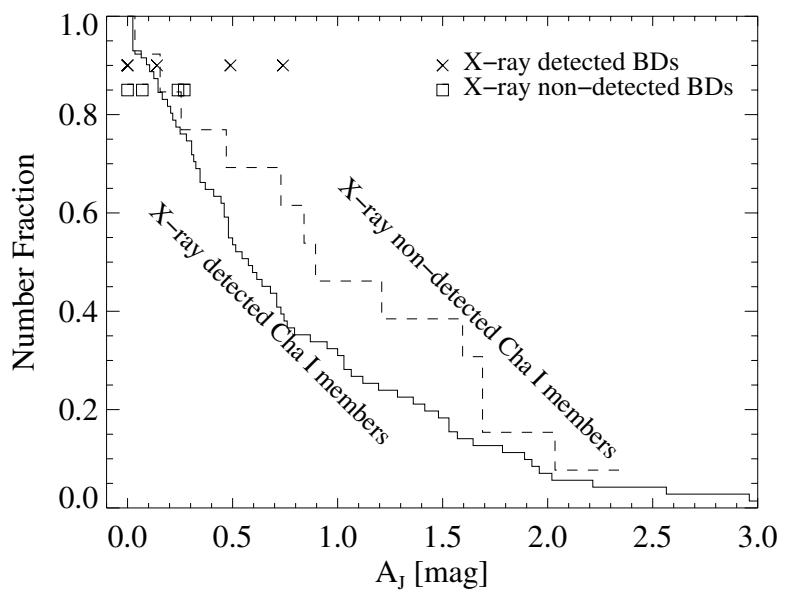

Fig. 6. Comparison of $J$ band extinction for X-ray detected (solid line) and X-ray undetected (dashed line) Cha I members; $A_{J}$ from Luhman (2004a). Substellar objects are plotted at their individual $A_{J}$ values and at an arbitrarily chosen fixed value on the vertical axis: Crosses are detected brown dwarfs, and squares undetected ones; substellar regime assumed to correspond to spectral type $\geq \mathrm{M} 6$.

$\mathrm{X}$-ray census in Cha I is complete down to this limit, at least in the area surveyed so far which comprises the central star forming sites.

For the Orion Nebula Cluster (ONC) it was shown that, next to intrinsically low X-ray flux, high extinction is one of the causes why even deep exposures may fail to register X-rays from (sub)stellar objects (Preibisch et al. 2005a). The cumulative number distribution of the $J$ band extinction, $A_{J}$, is shown in Fig. 6 for all X-ray observed Cha I members. The $A_{J}$-distribution of X-ray detections and non-detections is undistinguishable, as we verified in 2-sample tests implemented in $\mathrm{ASURV}^{6}$. In the sub-sample of probable brown dwarfs (spectral type $\geq$ M6) there is also no significant distinction between X-ray detected and undetected ones in terms of $A_{J}$, such that the non-detection of 4 brown dwarfs is probably due to their intrinsically weak emission.

As mentioned in Sect. 1, prior to the study presented here, only three bona-fide brown dwarfs of Cha I had been detected in X-rays: ChaH $\alpha 1$ and 7 (Stelzer et al. 2004) and CHSM-17173 (Robrade \& Schmitt 2007). We add here the X-ray detection of 2M $1101 \mathrm{~B}$ and possibly also 2M $1101 \mathrm{~A}$. With its spectral type of M8.25, 2M 1101 B is the coolest and least massive brown dwarf of Cha I detected in X-rays. Its X-ray luminosity is at the low end of all X-ray detected VLM objects in Cha I, and the upper limit for 2M $1101 \mathrm{~A}$ is lower than any of the detections.

Figure 7 shows the dependence of X-ray emission of the young members in Cha I on mass. The most important features in this diagram are completely consistent with findings in other star forming regions, in particular the ONC and Taurus, and can be summarized like this: (i) The well-known decline of $L_{\mathrm{x}}$ with decreasing mass is seen across the low-mass range (cf. Preibisch et al. 2005a; Telleschi et al. 2007, for the ONC and for Taurus, respectively), and this trend seems to continue into the brown dwarf regime (cf. Preibisch et al. 2005b; Grosso et al. 2007); (ii) there is no dependence of $L_{\mathrm{x}} / L_{\mathrm{bol}}$ on mass; (iii) for given mass a considerable (2-3 dex) spread in X-ray luminosity and $L_{\mathrm{x}} / L_{\mathrm{bol}}$ is observed. We recall, that the values shown in Fig. 7 are a collection of data obtained with different instruments

6 The Astrophysical SURVival analysis package is available at the astrostatistics site at Penn State University; see also Lavalley et al. (1992). 

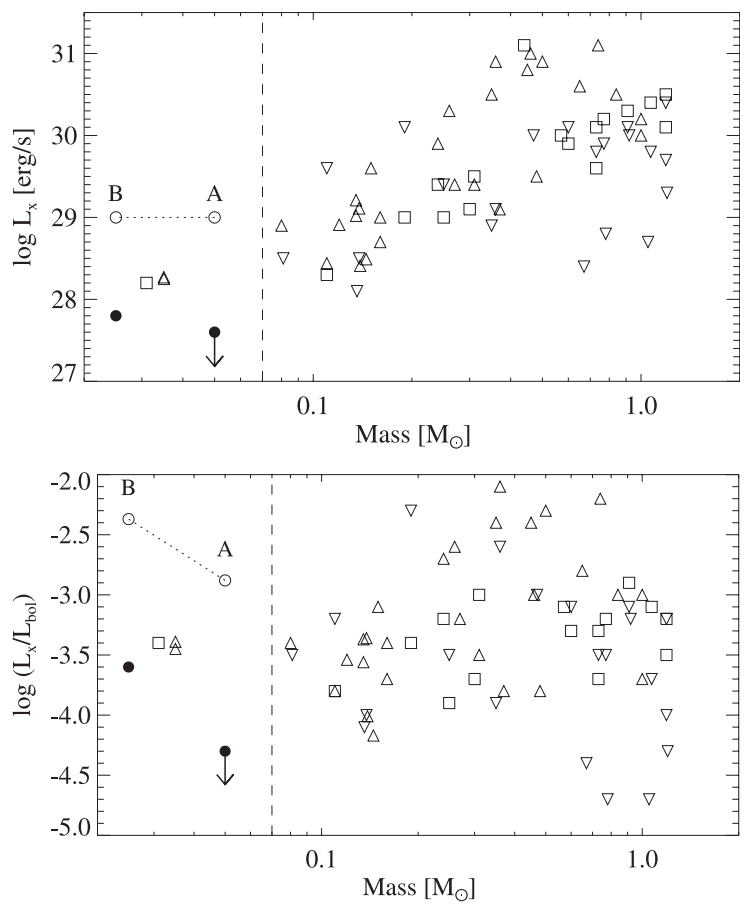

Fig. 7. X-ray luminosity versus mass for the low-mass Cha I members from the catalog of Luhman (2004a). For clarity no upper limits are shown. Upward triangles, downward triangles, and squares represent data from Stelzer et al. (2004), Feigelson \& Lawson (2004), and Robrade \& Schmitt (2007), respectively. For 2M 1101 A and B we show two positions: a) according to the XMM-Newton observation of the combined system 2M 1101 AB (open circles connected by dashed line to symbolize that the data is from one X-ray source), and $\mathbf{b}$ ) according to the Chandra observation in which the two components are resolved (filled circles). The dashed lines represent the substellar limit.

and different analysis techniques. Nevertheless, there seem not to be very significant systematic shifts of the X-ray luminosities measured in the three different studies. In particular, the fields analysed by Feigelson \& Lawson (2004) and Robrade \& Schmitt (2007) partially overlap. Most stars common to both fields show typical differences of a factor of two, which can be explained by the different energy bands in which the X-ray luminosity was measured: For a $1 \mathrm{keV}$ spectrum with $\log N_{\mathrm{H}}\left[\mathrm{cm}^{-2}\right] \approx 21.5$, the $0.5-8 \mathrm{keV}$ band used by Feigelson \& Lawson (2004) includes roughly half the flux of the $0.2-10 \mathrm{keV}$ band used by Robrade $\&$ Schmitt (2007). The X-ray fainter objects remain undetected in the XMM-Newton pointing, because at a similar effective exposure time of $\sim 70 \mathrm{ks}$ Chandra is more sensitive due to its low background. An extensive investigation of the X-ray census in Cha I is beyond the scope of this paper. Such a study is underway (Telleschi et al., in prep.), and we do not elaborate on this issue here.

In Fig. 8 the fractional X-ray luminosities of the VLM stars and brown dwarfs in Cha I are complemented with equivalent objects in Taurus and the ONC. Similar plots have been presented individually for the ONC (Preibisch et al. 2005a) and for Taurus (Grosso et al. 2007). The samples in these three star forming regions show that young substellar objects can emit as much X-rays as higher-mass $\mathrm{T}$ Tauri stars with respect to their bolometric luminosity, with the most active objects emitting near the saturation limit of $\log \left(L_{\mathrm{x}} / L_{\mathrm{bol}}\right) \sim-3$. In other words, the efficiency of the dynamo and the manifestation of activity phenomena show no break down to spectral type $\approx \mathrm{M} 8$ for objects of a few Myrs age. This is in agreement with recent

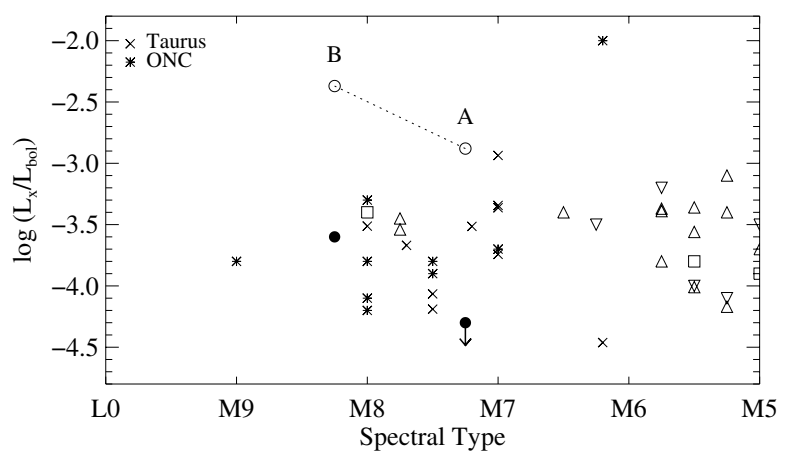

Fig. 8. Fractional X-ray luminosity versus spectral type for brown dwarfs of different star forming regions: Cha I - plotting symbols as in Fig. 7, Taurus - crosses (data from Grosso et al. 2007), and ONC asterisks (data from Preibisch et al. 2005b).

Table 4. Brown dwarfs and candidates (spectral type >M6) of Cha I observed with XMM-Newton and Chandra. Spectral type from Luhman (2004b), mass and age interpolated from Chabrier et al. (2000) models. According to Chabrier et al. (2000) the substellar limit is located at $\sim 0.07 M_{\odot}$, which corresponds to spectral type $\approx$ M6.5 (see also Fig. 1). The X-ray luminosity is from the reference given in the last column.

\begin{tabular}{llrrrl}
\hline \hline Designation & SpT & $\begin{array}{r}\text { Mass } \\
{\left[M_{\odot}\right]}\end{array}$ & $\begin{array}{r}\text { log Age } \\
{[\text { yrs }]}\end{array}$ & $\begin{array}{r}\log L_{\mathrm{x}} \\
{[\mathrm{erg} / \mathrm{s}]}\end{array}$ & Ref* \\
\hline 2M1101 B & M8.25 & 0.025 & 6.0 & 27.8 & this work \\
ChaH $\alpha$ 1 & M7.75 & 0.035 & $<6.0$ & 28.3 & S04 \\
ChaH $\alpha 7$ & M7.75 & 0.035 & $<6.0$ & 28.3 & S04 \\
CHSM 17173 & M8 & 0.041 & $<6.0$ & 28.5 & F04 \\
ESOH $\alpha 566$ & M6.5 & 0.08 & $<6.0$ & 28.2 & R07 \\
ISO-217 & M6.25 & 0.081 & 6.5 & 28.9 & S04 \\
\hline 2M1101 A & M7.25 & 0.05 & 6.0 & $<27.6$ & this work \\
ChaH $\alpha 11$ & M7.25 & 0.054 & 7.1 & - & S04 \\
ISO-138 & M6.5 & 0.068 & 7.0 & - & S04 \\
ChaH $\alpha 12$ & M6.5 & 0.074 & 6.4 & - & S04 \\
ChaH $\alpha 10$ & M6.25 & 0.074 & 6.9 & - & S04 \\
\hline
\end{tabular}

* References for X-ray luminosity: S04 - Stelzer et al. (2004),

F04 - Feigelson \& Lawson (2004), R07 - Robrade \& Schmitt (2007).

mean field dynamo models by Chabrier \& Küker (2006) that predict large-scale fields in fully convective objects, no matter whether they are stars or brown dwarfs, despite the absence of differential rotation in these objects. The X-ray observations of young brown dwarfs suggest, that the supposed decline of X-ray emission with decreasing atmospheric temperature due to the decoupling of plasma and field sets in at spectral types later than M8 for young ages, i.e. for $T_{\text {eff }}<\sim 2700 \mathrm{~K}$, consistent with the temperature range where the electrical resistivity is predicted to decline rapidly (Mohanty et al. 2002). Previous evidence for a connection between the X-ray emission level from brown dwarfs and $T_{\text {eff }}$ regarded more evolved ultracool dwarfs that have effective temperatures below $2500 \mathrm{~K}$ (Stelzer et al. 2006), and they present no contradiction to the picture drawn above for young substellar objects.

Despite the obvious ability to maintain high fractional X-ray luminosities, the X-ray detections of young brown dwarfs reported so far represent only the tip of the iceberg. The bonafide brown dwarfs in Cha I from published Chandra and/or XMM-Newton observations are summarized in Table 4. All Cha I brown dwarfs detected in X-rays so far are located on or above the $1 \mathrm{Myr}$ isochrone, and a similar tendency is obvious in Taurus and the $\mathrm{ONC}$, where mostly the very young, and consequently 
very luminous VLM stars and brown dwarfs have been detected in X-rays (Preibisch et al. 2005b; Grosso et al. 2007). With the exception of $2 \mathrm{M} 1101 \mathrm{~A}$, the undetected brown dwarfs have older ages on the Chabrier et al. (2000) tracks, i.e. lower bolometric luminosity. Since their upper limits to $L_{\mathrm{x}}$ are on average somewhat below the X-ray luminosity of the detections, they are expected to have $L_{\mathrm{x}} / L_{\text {bol }}$ levels near the range of the X-ray detected brown dwarfs. Therefore, deep X-ray pointings of lowluminosity young brown dwarfs will likely populate the $L_{\mathrm{x}} / L_{\mathrm{bol}}$ relation with a scatter similar to that observed for higher-mass pre-MS stars, while a shut off of X-ray emission may be expected for young brown dwarfs with temperatures below the ones observed so far.

Acknowledgements. We acknowledge financial support from ASI/INAF contract I/023/05/0. B.S. wishes to thank E. Flaccomio for stimulating discussions and the anonymous referee. The Chandra X-ray Observatory Center is operated by the Smithsonian Astrophysical Observatory for and on behalf of the NASA under contract NAS8-03060. XMM-Newton is an ESA science mission with instruments and contributions directly funded by ESA Member States and NASA.

\section{References}

Anders, E., \& Grevesse, N. 1989, Geochim. Cosmochim. Acta, 53, 197

Asplund, M., Grevesse, N., \& Sauval, A. J. 2005, in Cosmic Abundances as Records of Stellar Evolution and Nucleosynthesis, ed. T. G. Barnes, III \& F. N. Bash, ASP Conf. Ser., 336, 25

Baraffe, I., Chabrier, G., Allard, F., \& Hauschildt, P. H. 1998, A\&A, 337, 403

Bate, M. R., Bonnell, I. A., \& Bromm, V. 2002, MNRAS, 332, L65

Chabrier, G., \& Küker, M. 2006, A\&A, 446, 1027

Chabrier, G., Baraffe, I., Allard, F., \& Hauschildt, P. 2000, ApJ, 542, 464

Comerón, F., Neuhäuser, R., \& Kaas, A. A. 2000, A\&A, 359, 269

Comerón, F., Reipurth, B., Henry, A., \& Fernández, M. 2004, A\&A, 417, 583
Durney, B. R., De Young, D. S., \& Roxburgh, I. W. 1993, Sol. Phys., 145, 207 Feigelson, E. D., \& Lawson, W. A. 2004, ApJ, 614, 267

Feigelson, E. D., \& Montmerle, T. 1999, ARA\&A, 37, 363

Feigelson, E. D., Getman, K., Townsley, L., et al. 2005, ApJS, 160, 379

Freeman, P. E., Kashyap, V., Rosner, R., \& Lamb, D. Q. 2002, ApJS, 138, 185

Getman, K. V., Flaccomio, E., Broos, P. S., et al. 2005, ApJS, 160, 319

Grosso, N., Briggs, K., Güdel, M., et al. 2007, A\&A, 468, 391

Güdel, M. 2004, A\&A Rev., 12, 71

Hong, J., Schlegel, E. M., \& Grindlay, J. E. 2004, ApJ, 614, 508

Kraft, R. P., Burrows, D. N., \& Nousek, J. A. 1991, ApJ, 374, 344

Lavalley, M., Isobe, T., \& Feigelson, E. 1992, in Astronomical Data Analysis Software and Systems I, ed. D. M. Worrall, C. Biemesderfer, \& J. Barnes, ASP Conf. Ser. 25, 245

Luhman, K. L. 2004a, ApJ, 602, 816

Luhman, K. L. 2004b, ApJ, 614, 398

Luhman, K. L., Peterson, D. E., \& Megeath, S. T. 2004, ApJ, 617, 565

Maggio, A., Flaccomio, E., Favata, F., et al. 2007, ApJ, 660, 1462

Mohanty, S., Basri, G., Shu, F., Allard, F., \& Chabrier, G. 2002, ApJ, 571, 469

Neuhäuser, R., \& Comerón, F. 1998, Science, 282, 83

Preibisch, T., \& Zinnecker, H. 2002, AJ, 123, 1613

Preibisch, T., Kim, Y.-C., Favata, F., et al. 2005a, ApJS, 160, 401

Preibisch, T., McCaughrean, M. J., Grosso, N., et al. 2005b, ApJS, 160, 582

Rieke, G. H., \& Lebofsky, M. J. 1985, ApJ, 288, 618

Robrade, J., \& Schmitt, J. H. M. M. 2007, A\&A, 461, 669

Rosner, R., Golub, L., \& Vaiana, G. S. 1985, ARA\&A, 23, 413

Smith, R. K., Brickhouse, N. S., Liedahl, D. A., \& Raymond, J. C. 2001, ApJ, 556, L91

Stelzer, B., Micela, G., \& Neuhäuser, R. 2004, A\&A, 423, 1029

Stelzer, B., Micela, G., Flaccomio, E., Neuhäuser, R., \& Jayawardhana, R. 2006, A\&A, 448, 293

Telleschi, A., Güdel, M., Briggs, K. R., Audard, M., \& Palla, F. 2007, A\&A, 468, 425

Vuong, M. H., Montmerle, T., Grosso, N., et al. 2003, A\&A, 408, 581

Weisskopf, M. C., Brinkman, B., Canizares, C., et al. 2002, PASP, 114, 1

Whittet, D. C. B., Prusti, T., Franco, G. A. P., et al. 1997, A\&A, 327, 1194

Wichmann, R., Bastian, U., Krautter, J., Jankovics, I., \& Rucinski, S. M. 1998, MNRAS, 301, L39 